\title{
Active OB Stars - an introduction
}

\author{
Dietrich Baade ${ }^{1}$, Thomas Rivinius ${ }^{2}$, Stanislas Stefl $^{2}$, and \\ Christophe Martayan ${ }^{2}$ \\ ${ }^{1}$ European Organisation for Astronomical Research in the Southern Hemisphere, \\ Karl-Schwarzschild-Str. 2, 85748 Garching. b. München, Germany \\ email: dbaade@eso.org \\ ${ }^{2}$ European Organisation for Astronomical Research in the Southern Hemisphere, \\ Casilla 19001, Santiago 19, Chile \\ email: triviniu@eso.org, sstefl@eso.org, and cmartaya@eso.org
}

\begin{abstract}
Identifying seven activities and activity-carrying properties and nine classes of Active OB Stars, the OB Star Activity Matrix is constructed to map the parameter space. On its basis, the occurrence and appearance of the main activities are described as a function of stellar class. Attention is also paid to selected combinations of activities with classes of Active OB Stars. Current issues are identified and suggestions are developed for future work and strategies.
\end{abstract}

Keywords. stars: activity, stars: binaries, stars: circumstellar matter, stars: early-type, stars: emission-line, Be, stars: magnetic fields, stars: mass loss, stars: oscillations, stars: rotation

\section{Active OB Stars: the concept}

\subsection{The activities}

The term Active B Stars was introduced in 1994 when the IAU Working Group on Be Stars was renamed Working Group on Active B Stars. The name was to capture all physical processes that might be active in Be stars and so be required to understand Be and other active B stars, similar to Richard Thomas' standing characterization of Be stars as the crossroads of OB stars. This paper considers every intrinsically variable OB star an Active OB Star.

The potential (and actual) variabilities are the same as everywhere else in the Hertzsprung-Russel Diagram (HRD): Stars may pulsate and so vary in temperature and shape. If they possess a magnetic field, there may be associated activity, and rotation will periodically modulate observables, especially if diffusion has led to locally varying surface abundances and/or the magnetic field is strong enough to trap an otherwise present wind. Very young stars may still be working on the dispersal of their natal disk, and somewhat more evolved stars may return some of the originally accreted mass to the ambient space through radiatively driven winds and/or discrete mass-loss events. Both fossil and newly lost circumstellar matter may cause variable extinction. The associated large-scale dynamics as well as local variations in temperature, density or excitation would manifest themselves also in variable profiles of spectral lines with circumstellar origin.

\subsection{The stellar classes}

With the objective of merely providing a first crude chart of the Active OB Star territory, this section tries to use the smallest amount of information necessary. All in all, this territory is presently divided into nine major, multiply overlapping areas (see Fig. 1).

At both very young and advanced ages, OB stars are variable so that they are Active OB Stars on account of their evolutionary phase. They are then respectively known as Herbig Be stars and supergiants or even Luminous Blue Variables (LBVs). 


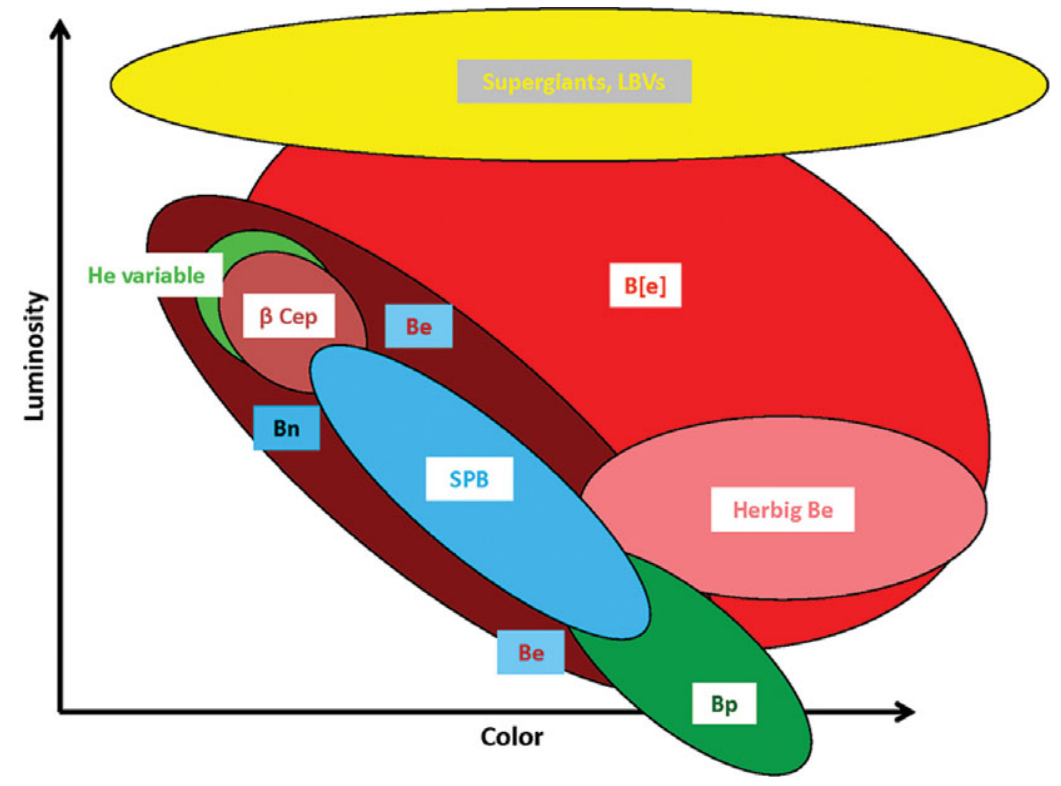

Figure 1. Schematic HRD of the various classes of Active OB Stars. The B[e] blob would be grossly oversized if $\mathrm{B}[\mathrm{e}]$ stars did not include pre-main sequence and post-main sequence stars but just the latter. Within a couple of years, major revisions can be expected from much expanded observing efforts.

Two classes of Active B Stars are defined primarily by the presence and appearance of circumstellar emission lines: Be stars (sometimes also dubbed "classical Be stars") and $\mathbf{B}[\mathbf{e}]$ stars. Be stars are characterized by rapid rotation. The appearance of their emission lines depends strongly on aspect angle, implying that the circumstellar matter, from which they arise, forms rotationally flattened equatorial structures. Be stars are further distinguished from $\mathrm{B}[\mathrm{e}]$ stars by not having any appreciable amounts of circumstellar dust while many B $[\mathrm{e}]$ stars exhibit infrared excesses that can only be explained by warm dust. The notation [e] indicates that forbidden low-excitation emission lines are common in $\mathrm{B}[\mathrm{e}]$ stars. But such lines are not a mandatory attribute of the class.

More work is needed to find out whether the classes of Be and $B[e]$ stars require further subdivision. This is especially true for the $\mathrm{B}[\mathrm{e}]$ stars, which have been identified among pre-main sequence stars as well as supergiants whereas more recent work has questioned very young ages of $\mathrm{B}[\mathrm{e}]$ stars. Among the Be stars, the later spectral sub-classes are often said to be less active on all time scales.

Be stars have somewhat inconspicuous cousins, namely rapidly rotating B stars not known to have shown optical line emission. For the lack of the latter, and not considering interferometric techniques, such stars cannot be recognized unless they are viewed roughly equator on and reveal their extremely broad spectral lines. In the MK notation they are known as Bn or Bnn stars. Unlike Be stars, they do not seem to pulsate in loworder $(\ell=|m|=2)$ nonradial pulsation (NRP) modes while higher-order modes with $m \sim$ 10 appear common. However, this distinction is still pending confirmation from larger samples, which should also examine whether there is any variation with spectral type.

There are two classes of Active OB Stars that have no defining properties other than their pulsations: The classical $\beta$ Cephei stars between B0 and B2 and the Slowly Pulsating B (SPB) stars between B2 and B7. Both types occur along the main sequence and actually overlap around B2, with the two domains being properly delineated only 
now by space observatories such as BRITE, CoRoT, Kepler or MOST. More disjunct seem to be the ranges in periods: $0.1-0.25 \mathrm{~d}$ for $\beta$ Cep stars but $0.5-3 \mathrm{~d}$ for SPB stars.

Magnetic OB stars, too, come in a hot and a cool variety: the helium-variable stars $\leqslant$ B2 and the Bp stars $\geqslant$ B7. Typical longitudinal field strengths are around several $\mathrm{kG}$. They are most easily recognized through rotationally propagating surface abundance anomalies, which at the hot end mostly involve helium and rare earths at the cool one and may artificially widen, or even create, the gap in spectral type. Of?p stars and $\tau$ Sco-like stars present further magnetic sub-groups.

Various kinds of activities can easily co-exist. In the HRD, more or less the entire area apportioned to OB stars is covered by one or more Active OB Star tiles. However, these tiles are porous because Active OB Stars are only an estimated 5-25\% minority. With ever more sensitive observations, this fraction keeps increasing.

The expected wealth of new observations is likely to demand major revisions, especially of the definitions of B[e], pulsating, and magnetic stars. Some activities extend over larger regions than the quoted spectral-type based names suggest: In addition to Be stars, there are Oe and Ae stars although the latter mostly manifest themselves through narrow absorption lines when the line of sight passes through the disk. Bp stars continue into the A-types domain, and there are yellow supergiants. Herbig Ae stars are more numerous than Herbig Be stars. Concerning pulsations, the boundary region between radiative and convective envelopes, which on the main sequence is not far from the A/B subdivision in spectral type, should let stars on either side have their own pulsation properties.

\section{The OB-Star Activity Matrix}

From the above compilations of variables and properties, the OB-Star Activity Matrix can be constructed. Fig. 2 shows it filled with what is believed to be today's knowledge. Most of the rest of this paper consists of a discussion (and sometimes a justification) of the contents of the Activity Matrix. This is partly organized in an 'across'- and 'down'like manner, with more emphasis on the vertical dimension because it is the one less often explored as most (especially observational) studies are object oriented. Finally, various combinations of links between selected matrix elements are examined.

\section{Analytical tools and models}

The past two decades have seen progress in the amount and quality of observations that would have been difficult to predict. But the understanding of Active OB Stars has probably benefited still more strongly from the vast improvements in the flexibility of models, which now affords their tailoring to specific observations. Also, while just 10 years ago most model calculations would assume slow rotation at most, the effects of extreme rotation are now widely considered.

Within the suite of models, stellar evolution assumes a central position because it is the one that puts all else into a common perspective. It does not stop at the level of single objects but also quantifies the chemical, radiative, and mechanical output of OB-star populations, which often dominate the corresponding global balances of their environments up to entire galaxies. The interface to explosive models terminating the evolution of many OB-stars is similarly important.

Evolutionary and mass-loss models continue to intensify their links. Mass loss from massive stars can exceed $50 \%$ in the first few million years with drastic effects on evolution. Radiative-wind models incorporate more and more of the observations that picture 


\begin{tabular}{|c|c|c|c|c|c|c|c|}
\hline & Pulsation & $\begin{array}{l}\text { Magnetic } \\
\text { field }\end{array}$ & Wind & $\begin{array}{l}\text { Mass loss } \\
\text { events }\end{array}$ & CSM & $\begin{array}{l}\text { Very fast } \\
\text { rotation }\end{array}$ & $\begin{array}{l}\text { Dependencies } \\
\text { on metallicity }\end{array}$ \\
\hline $\begin{array}{l}\text { Supergiants, } \\
\text { LBVs }\end{array}$ & (X) & (X) & $\mathrm{x}$ & (X) & $x$ & $(\mathrm{X})$ & $\mathrm{x}$ \\
\hline $\mathrm{B}[\mathrm{e}]$ stars & & & $(X)$ & & $x$ & & \\
\hline Be stars & $x$ & (X) & $((\mathrm{X}))$ & $x$ & $x$ & $x$ & $x$ \\
\hline Bn stars & $x$ & & & & & $x$ & \\
\hline $\begin{array}{l}\beta \text { Cephei } \\
\text { stars }\end{array}$ & $x$ & (X) & (X) & & (X) & & \\
\hline SPB stars & $x$ & $(\mathrm{X})$ & & & & & \\
\hline $\begin{array}{l}\text { He-variable } \\
\text { stars }\end{array}$ & & $x$ & $x$ & & $x$ & (X) & \\
\hline Bp stars & & $x$ & & & & & \\
\hline $\begin{array}{l}\text { Herbig Be } \\
\text { stars }\end{array}$ & & $(\mathrm{X})$ & $x$ & & $x$ & & \\
\hline
\end{tabular}

Figure 2. The OB-Star Activity Matrix. Crosses: combinations of property and type of star that are typical. Single brackets: the combination is not generally established. Double brackets: doubtful cases. Not marked: combinations do not exist or are not known to exist.

mass loss from OB stars as a very dynamic, sometimes violent process. Not any less important are smaller-scale clumps. The increased escape probabilities for photons between such clumps has led to downward revisions of mass loss rates by factors of $\sim 5$.

Evolution models have especially advanced in the handling of mixing processes so that relatively subtle surface abundance patterns can be predicted, which may encapsulate much of a star's history. This strongly broadens the practical scope of stellar atmosphere and radiation-transport models that have been developed to ever higher perfection.

Asteroseismology has been around as a term for a long time. With the coming into operation of wide-angle space photometers many of the promises will now come true for many types of Active OB Stars - as the first results suggest, after much additional work. The eventual combination of stellar evolution models with surface abundances and asteroseismologically measured internal structures stands high chances to lay the most important foundation to the understanding of Active OB Stars.

Another application of complex data inversion techniques is the incipient construction of images from interferometric observations with multiple telescopes and baselines.

OB stars shape their environments through winds and radiation and partly build their own circumstellar structures, e.g., through discrete mass-loss events (LBVs, Be stars) or in the form of magnetospheres. Much of this is becoming accessible to interferometers and so demands radiative and dynamical models to be combined and extended to higher spatial dimensionality. The current rate of progress is impressively high.

Much of this work has been enabled not just by ever cheaper hardware but especially by the parallelization of codes that now often run on hundreds of CPUs. The partial replacement of CPUs by Graphics Processing Units is opening another new phase.

\section{Binaries and binarity}

The Activity Matrix lists neither binaries as a distinct class of Active OB Stars nor binarity as a distinct type of intrinsic variability. However, it is important to recall what situations can arise in double stars that are not likely in single stars. 
Most prominent to name are binaries, in which the primary has entered one of the classes of Active OB Stars as the result of binary evolution. For instance, some Be stars have hot sub-dwarf companions and probably owe their fast rotation to mass transfer from the companion.

The orbital motion and associated modification of the gravitational potential may trigger single events as well as trigger or suppress long-term behaviors. For instance, the ejection of a circumstellar disk around the primary in $\delta$ Sco has been tentatively attributed to the periastron passage. It is hoped that the next periastron passage in 2011 will clarify this issue. If the disk ejection is indeed caused by the varying gravitational pull, one may wonder whether the transmitter process is forced nonradial pulsation. On the other hand, $\beta$ Cephei stars seem to be rare in, or even absent from, binaries with orbital periods of less than a couple of days.

If an $\mathrm{OB}$ star with circumstellar structures is orbited by a compact object, periodic dives of the companion through that matter ideally probe its properties. Conversely, the response of the latter can reveal much detail about the nature especially of exotic objects; but there are also effects on the large-scale dynamics, e.g., truncation or precession of disks. In other OB binaries, both stars may have a wind, the collision of which establishes a similarly interesting laboratory.

Last but not least, there are also special features like the jet in $\beta$ Lyrae, which deserve attention because of their seemingly rare occurrence. But it may also happen that a star appears very exceptional only as long as the role of a companion has not been appreciated. For instance, $\beta$ Cep was claimed to break the rule that all Be stars rotate rapidly, until it was realized that the emission lines actually belong to a nearby second star.

The most important property of binaries is, of course, their enabling role in the quantitative measurement of fundamental stellar parameters. Because activities will increase the noise at least of the measurements, many Active OB Stars cannot be primary sources of such information. But comparisons with not-active OB stars will still be essential.

Since the fraction of binaries is high among early-type stars, the possibility of some observed properties being due to binarity must always be considered. But there does not seem to be any intrinsic variability limited to binaries, and there is no obvious class of Active OB Stars with much above-average binarity rate.

\section{Activities as a function of stellar class}

\subsection{Pulsation}

Of all activities observed in Active OB Stars, pulsation is the most frequently and widely detected one. From ground-based observations, which were limited in number and especially sensitivity, it seemed that $\beta$ Cep stars pulsate in nonradial $p$ modes and often also radially, Be stars in $g$ - as well as in $p$-modes, SPB stars in $g$-modes, and Bn stars in $p$-modes. Supergiants were mostly suspected to pulsate in $g$-modes with $p$-modes occasionally being a possibility.

With the advent of space-borne wide-field precision photometry, this chapter in the history of Active OB Stars is presently undergoing so strong and rapid revisions that it is advisable to neither repeat outdated past wisdom nor speculate about unconfirmed new insights. The still relatively few pulsation spectra published so far are partly very rich and do not in all cases find straight-forward matches in theoretical models, especially in the presence of very fast rotation. Also the mapping of the occurrence of various pulsational properties has seriously commenced only with these observations. 
An interesting question that has not been addressed for a fair while is: High- $m$ nonradial modes are only seen in broad-lined stars. Is this so because such modes are difficult to detect in narrow lines or because rapid rotation favors their excitation?

Another theoretical expectation that is still awaiting attempts of an observational verification is that nonradial $g$-modes may transport angular momentum between different radial zones of a star, with possible evolutionary ramifications.

\subsection{Magnetism}

On average, magnetic fields are more difficult to detect than pulsations are, especially on small spatial scales, so that magnetism might actually be the premiere activity of Active OB Stars. Detections have been reported for almost any kind of OB stars; increases in the sensitivity and diversity of searches may wash out the current classification scheme and propose new criteria. But some reports have provoked strong responses questioning claimed significances. Unfortunately, the current plans for ELTs do not have polarimetry among their main science drivers so that studies of magnetic fields (and other applications of spectropolarimetry) may not benefit from the large gain in light-collecting power.

Because magnetic fields below the current detection threshold of 50-100 G for the mean field strength may still have significant dynamical effects (and thereby make convenient attributes of various toy models), methods other than polarimetry may have to be resorted to. For instance, it is conceivable that space photometry may place constraints on the strength and structure of magnetic fields: If some variability can be attributed to rotation, star spots are a reasonable guess for the explanation, and the associated light curve would give hints at their size, contrast, and latitude. Non-periodic variations would require still more interpretative caution but could also be related to magnetic activity.

Although the rich spectrum of observations demonstrates that actual OB stars do not generally find it difficult to exhibit strong, large-scale magnetic fields, theoretical 'confirmation' is missing. In fact, there may be no other topic in Active OB Star research, where observations and models are farther apart from one another although only two basic mechanisms seem to require consideration: dynamo processes and the freezing in of magnetic fields present in the parental cloud. Although sufficiently effective dynamos are difficult to develop in mostly radiative envelopes, the clustering of magnetic stars along the main sequence may give this hypothesis a slight edge over the fossil alternative. Interesting may also be the recent report that, in Bp stars, magnetic fields only become detectable after $30 \%$ of the main-sequence evolution has been completed.

\subsection{Structured winds: Photospheric heritage?}

Observations find more and more structure in the mass loss from OB stars. Extended IUE campaigns have established a long time ago that Discrete Absorption Components (DACs) in wind lines often repeat on rotational time scales. The concept of Corotating Interaction Regions has enabled models to reproduce the observations very well. However, the origin and nature of the perturbations at the base of the wind have remained unidentified. The velocity fields and temperature modulations of the wide-spread nonradial pulsations are obvious candidates. With very few exceptions, observations have been insufficient to demonstrate a satisfactory match of the time scales, let alone periods. Perhaps, long-term photometry from space can contribute further cases.

Large-scale magnetic field structures would a priori lead to rotational modulations (if any). In OB stars with unambiguously detected magnetic fields, periodic effects on the wind can be clearly seen. If the field strength reaches $\mathrm{kG}$ levels, the wind is trapped in a magnetosphere. However, magnetic fields are not so far generally detected; indirect support may come from space-based precision photometry. 
Groundbased time-resolved spectropolarimetry of emission lines has identified irregularly outward-moving knots, which have led to the concept of porous winds with embedded clumps. Unlike DACs, these small-scale structures significantly affect the escape probability of wind-driving photons, reducing mass loss rates by a factor of $\sim 5$.

Inspired by the case of Be stars, equatorial density enhancements have been searched for also in rapidly rotating $\mathrm{O}$ stars. The results were mostly negative. Therefore, disk formation in the more extremely rotating Be stars may not just be a property of winds in the presence of very fast rotation, as various models have tried to picture. In particular, the angular momentum necessary for rotationally supported disks is not supplied by a wind alone. So far, this is only achieved by viscous decretion. This model has the additional important strength that it also explains the subsequent angular momentum transport from the inner to the outer disk.

Once formed, disks of Be stars only last for $\sim 10$ years. Observations show that disk dispersal progresses inside-out so that stellar radiation pressure may be involved in this process. In fact, insufficiently sampled and coordinated ground-based and IUE observations suggest that the strength or even presence of winds from Be stars may depend on the presence of a disk: Winds only set in after a significant disk has been built up, and they cease before the optical line emission from the disk has fully vanished. Alternatively, if during active phases disk replenishments were frequent, the wind might be fed with debris left over from this process. Either ansatz might also explain the puzzling fact that, in Be stars, stellar winds are observed down to cooler spectral types than in non-Be stars. More importantly, it may be useful to identify those wind variabilities that Be and other OB stars have in common. Although (early-type) Be stars exhibit strong nonradial pulsations, the latter may not be an important source of wind variability.

$I U E$ observations also suggest that the winds of Be stars are strongest at intermediate inclinations and, therefore, stellar latitudes. This is consistent with the wind material being supplied by the disk-building process. By contrast, recent interferometric observations of Be stars report polar structures that are being identified as winds. In the absence of velocity information, this latter step seems to be a very large on.

\section{Metallicity}

In Table 2, column "Dependencies on metallicity" is special in that it actually presents a third dimension, which reports whether the frequency or properties of a class of Active OB Stars have been observed to depend on metallicity. Metallicity is, therefore, perhaps the most valuable diagnostic because a model is vastly more convincing if it reproduces observations over a range in metallicity. This makes it important to take advantage of the present 8-10 m class telescopes and the planned generation of ELTs, which bring the Milky Way's satellite galaxies and their metallicity levels within the reach of fairly high spectral resolution. Since at the same time the number of stars in such galaxies is larger per unit area in the sky, multi-object spectroscopy can be used to good advantage.

Low metallicity reduces the efficiency of the linear-momentum transfer from radiation to matter. The resulting basic dependency of mass loss rates on metallicity is well established in luminous stars, which are observable even beyond the local group.

The accompanying reduced loss of angular momentum is one reason to expect lowmetallicity stars to rotate more rapidly, with the other reason being that at lower opacity stars have smaller radii if all else is equal. Both circumstances imply a higher fractional critical rotation rate. On this ground, it has been argued that, because of their rapid rotation, Be stars should be more frequent in low-metallicity environments, which is, in fact, observed. However, in a strict sense, this does not permit the sometimes heard 
conclusion to be drawn that low metallicity makes more Be stars. Rapid rotation only is a necessary condition for the Be phenomenon; according to all what is known today, rotation alone is not sufficient for a B star to become a Be star: In the Galaxy, the number of Bn stars is about as large as the one of Be stars. Therefore, one can only claim that, down to SMC metallicity levels, whatever other properties are needed for the Be phenomenon, their net dependency on metallicity is not strongly negative. This also leads to the following: It has been demonstrated that the beating of nonradial pulsation modes can drive mass loss events in Galactic Be stars. But pulsation responds negatively to reduced metallicity so that one would expect such a mechanism to be less effective in the SMC. Since the SMC is more abundant in Be stars than the Galaxy, this might be another hint that $\omega / \omega_{c}$ increases at low metallicity.

The prediction by theory of a metallicity dependence of pulsations is still without observational counterpart for comparison. And the sensitivity or not to metallicity of dynamo processes or fossil magnetic fields is hardly explored at all.

\section{Classical disputes about classical Be stars}

Previous meetings with a large fraction of Be star contents sometimes became quite lively on the grounds of widely differing opinions. Today, most of these issues can be considered closed. Others have arisen but are discussed less sanguinely:

- Can low-velocity, low-excitation and high-velocity, high-excitation spectral lines be put into one common radial scheme? No, they cannot. There is a cool, geometrically thin disk with a fast and hot wind at higher latitudes.

- Can transitions (observed in a few stars) from a Be to a Be-shell phase (with narrow absorption lines sumperimposed to very broad emission lines) and back to a Be phase (without such additional absorption lines) be explained by a radial restructuring of the atmosphere? No, they cannot. The most plausible model involves disk precession in a binary star. In single stars, warping or flaring of the disk are alternatives.

- Is the photospheric line-profile variability due to rotation or to nonradial pulsation? It could be both with pulsation being the dominant effect by far. Not surprisingly, space photometry finds evidence also of rotation time scales. But the cross identification in line profile variations is still pending.

- Are the variations in the violet-to-red ratio of the strength of emission line components due to orbital aspect variations? No, they are not. They are the signature of global, onearmed oscillations of a Keplerian disk around a rotationally distorted star.

- Are all Be stars products of binary evolution? Yes, some are. But there is no evidence of an overabundance of binaries among Be stars. (If they were even underabundant one might be driven to wonder whether the rapid rotation comes about because the initial angular momentum does not have to be shared with the orbital motion of a companion.)

- Is rotationally induced polar enhancement the reason that Be stars exhibit winds down to later spectral types than do normal B-type stars? It depends. At the same polar $T_{\text {eff }}$ of a rotating and a non-rotating star, the difference is small for pole-on stars. At other viewing angles, the comparison is made difficult by the range in $T_{\text {eff }}$ of the rapid rotator. The IUE database appears more consistent with the wind being fed by radiative ablation of the circumstellar disk (cf. Sect. 5.3).

\section{Selected combinations of specific properties}

\subsection{Rotation + pulsation + mass loss (events) in Bn/Be stars}

For every broad-lined Be star, the Bright Star Catalogue contains between 0.5 and 2 Bn stars with about the same spectral type and magnitude. In view of the 'saturation' in 
rapidly rotating stars of conventionally used optical lines to measure rotation velocities, it is not certain that $v \sin i$ values measure the same thing in Be and Bn stars. But it is reasonable to suspect that the differences are not dramatic (even though potentially systematic, also because of circumstellar contaminations in Be stars).

While (early-type) Be stars pulsate in low-order $g$-modes and higher-order $p$-modes, only the latter seem to be present in Bn stars. At the same time, only Be stars develop circumstellar disks whereas Bn stars do not exhibit emission lines. This invites the suspicion that the low-order modes play a role in the disk-building process. It is supported by two empirical facts: (i) The variability of optical emission lines suggests that individual events may dominate the mass loss from Be stars. (ii) In a very small number of Be stars evidence has been found that such events are driven by the beating of nonradial pulsation modes with the same $\ell, m$ values.

However, reality is more complex: More common are Be stars, in which intensive longterm spectroscopic monitoring could not find a second low-order mode. And yet, these Be stars do undergo outbursts. If Be stars rotate close to the critical rate, outbursts not assisted by beating NRP modes pose no fundamental problem because any process with associated velocities of the order of the turbulence could let the total velocity exceed the critical threshold. An alternative, which existing observations may not be able to address fully, is that in such stars the beat periods are of the order of some years, i.e., the time between consecutive outbursts, which can be relatively equally spaced. In order to study this matter more broadly, photometric databases such as OGLE or MACHO could be searched for Be stars with regularly spaced outbursts. Since the primary pulsation frequency normally is easily found, the difference between this frequency and the one of the outbursts would correspond to a second pulsation frequency to be searched for - if the outburst frequency equals a beat frequency.

The outbursts of Be stars are reminiscent of classical limit-cycle systems: Some mechanism drives the star quasi-peridocially to the limit of stability. It is the beating of NRP modes in some cases, but may also be angular-momentum transfer from the core to the envelope. The angular-momentum redistribution, too, may be caused by NRP modes (which, however, should be prograde), or it may be accomplished by magnetic fields, meridional circulation, other processes or any combination thereof. There is no reason to believe that only one mechanism converts Bn stars to Be stars. The mix of such processes may depend on mass; ditto for the threshold in $\omega / \omega_{c}$. In fact, it has been reported that the fractional critical velocity required for the Be phenomenon increases towards lower masses. The Bn-to-Be number ratio increases in the same direction, which is a consistent observation but requires cautious interpretion becauses cooler stars are less effective in ionizing disks, which may, then, remain undetected because of weak line emission.

\subsection{Magnetic fields + rotation}

Magnetic fields brake the rotation of young convective stars. Consistent with that, Bp stars are throughout slow rotators. Therefore, sometimes the reasoning is inverted, and it is argued that rapidly rotating stars, e.g., Be stars, do not possess significant magnetic fields. The logic of this conclusion is impeccable: If A implies B, not-B implies notA. However, recently a new population of extremely rapidly (nearly critically) rotating helium-variable stars was discovered. Perhaps, their evolutionary history is at least as exciting as their present properties. The contradiction between rapid rotation and strong magnetic field could be resolved if either or both properties had been acquired only during the course of time. The reported delayed occurrence of magnetic fields in Bp stars could be interesting also in this context. 


\subsection{Pulsations + magnetic fields}

Pulsations and magnetic fields co-exist in various Active OB Stars. Interestingly, there seem to be no known combinations of kGauss fields with pulsation. This may be an issue of observational sensitivity. But it could also result from magnetic fields inhibiting the equalization on spherical surfaces of opacity, mean molecular weight, etc. It would be very attractive to investigate strongly magnetic stars for pulsations from space.

Magnetic fields at a level of up to a few 100 Gauss have been reported for a number of $\beta$ Cep as well as SPB stars. But there are no deep enough seismological analyzes that would permit the detection of any magnetic effects on the pulsations. There has been the suggestion that, similar to the rapidly rotating Ap stars, $\beta$ Cep might be an oblique magnetic rotator, in which the pulsational symmetry axis is aligned with the magnetic rather than the rotation axis. Because this report has remained without independent confirmation, also in other stars, it may be uncertain. (But magnetic axes seem to be mostly tilted with respect to the rotation axes.)

\section{The fate of Active OB Stars}

Can any terminal evolutionary stages of stars be traced back to specific types of Active OB Stars? It seems fairly certain that Bp stars evolve to magnetic white dwarfs. There is also ample evidence that stars with initial masses above $\sim 8 M_{\odot}$ end up as core-collapse supernovae, but already the nature and temporal sequence of the intermediate stages (LBVs and the various flavors of Wolf-Rayet stars) still give rise to discussion.

The collapsar model is widely supported as one explanation of long Gamma-Ray Bursters (GRBs). The required rapid rotation renders Oe/Be stars with initial masses above $20 M_{\odot}$ interesting candidates. But rapid rotation may also result from mass transfer in binaries. In either case, low metallicity would help to maximally preserve the accumulated angular momentum. In fact, evidence is emerging that low-metallicity environments do favor GRB events.

Still more uncertain is it whether (rapidly rotating) magnetic helium-variable stars play a role as progenitors of magnetars and Soft Gamma Repeaters. The small number of known helium-variable stars is a serious obstacle.

\section{A wish list for future work}

The progress in the understanding of Active OB Stars is driven through many channels, and the highest priority must be to preserve and enlarge this diversity. Still, there are some developments that may have a particularly broad impact:

- Hot stars emit most of their flux in the UV. The variability of Active OB Stars requires monitoring on many time scales, which is not easily done with present large space-borne facilities. But an IUE-like satellite that is small enough to be of no use outside the Local Group could close one of the largest gaps in the arsenal of observing facilities. Since many of the $I U E$ observations were interpreted on the basis of the understanding of 20 years ago, a first valuable step could consist of a re-analysis of pertinent archival data.

- Non-active members of star clusters present the most reliable basis for the study of Active OB Stars because distances, ages, metallicities, etc. can be derived with good accuracy. Only statistics obtained from star clusters can empirically guide stellar evolution models. Star clusters may also help identify the switches that do or do not let OB stars branch off main-track evolution towards the various types of Active OB stars. Much of the existing work has been done using imaging instruments. But multi-object spectroscopy, or at least slit-less spectroscopy, obviously has a much higher potential. Pure 
photometry reaches hard limits if observations of extreme rotators are not corrected for gravity darkening. However, photometry alone cannot determine $v_{e q}$ and $i$.

- Recent years have seen spectacular progress from interferometric observations of Active OB Stars. The potential of such work is very far from having been exhausted. Spectrointerferometry and first simple images from closure phase techniques show the way.

- The 'poor man's substitute for interferometry' is (spectro-)polarimetry, which sometimes can yield nearly equivalent insights. Regrettably, it is broadly underutilized.

- Satellites suitable for asteroseismology (e.g., BRITE, CoRoT, Kepler, MOST) have already produced amazing results for selected stars. Broader statistics is very much wanted. (Like searches for extra-solar planets, with which such facilities are often shared, statistics benefits from relatively large telescope apertures. However, this limits the maximal brightness of observable targets and so excludes objects, for which the also needed long series of spectral observations are available and/or feasible.)

With numerical models moving ever closer to reproducing individual Active OB Stars, also the reverse becomes increasingly more true: Observations without advanced modeling support are losing in value.

\section{Outlook}

Maybe, in 25+ years, there will be an IAU Symposium Active OB Stars Explained!. The following attempts to give a selective preview of the agenda and work it may be based on; the admitted Be-centricity may prove to be extendable to other classes of Active OB stars:

- The difference(s) between Be and Bn stars: Their dominating pulsation modes seem to be different. Therefore, seismology should be the method of choice.

- The evolutionary stage of Be stars: Since the dominating NRP modes excited in (early-type) Be stars seem to be deep-reaching $g$-modes, they may hold one key to the answer, depending on the number of modes found by space photometry. Since at the length of Be-star periods frequency spectra are dense, sufficiently precise identifications may be challenging so that cross-calibration with open-cluster data could be important. - The threshold of $v_{e q} / v_{\text {crit }}$ for the Be phenomenon: This will reveal how important the contributions by other processes to the release of energy and angular momentum need to be. A pre-requisite is the determination of the inclination angle, $i$, from precision photometry (direct rotational modulation? rotational splitting of NRP frequencies?), photospheric line-profile modeling (taking into account rotational gravity darkening and rotationally modified angular NRP eigenfunctions), fitting of circumstellar emission lines, and possibly also interferometry. For comparison, $v_{e q} / v_{c r i t}$ needs to be determined for a number of Bn stars as well (which have large values of $i$ by definition). Asteroseismology will also show whether extreme rotators spin differentially.

- The role of $\ell, m=2,+2 g$-modes in the mass loss from Be stars: Results for the previous point will be valuable also in this context. But (the already beginning) observations of the behavior of pulsational frequencies and amplitudes during outbursts will provide the key. The main technical challenge will be the proper timing of such observations. Stars with mass loss events triggered by the beating of pulsations modes and stars with frequent mass ejections should be the first targets. However, the analysis must remember the selection biases associated with such a strategy.

- The relevance of magnetic fields for the Be phenomenon: Since conventional spectropolarimetry seems to have reached its limits without delivering a significant number of detections, the technique might have to team up with interferometry (at baselines of several $100 \mathrm{~m}$ ) to search for smaller-scale structures. Candidates might result from 
high-precision photometry. High-energy phenomena associated with magnetic processes might provide an additional avenue.

- The fraction of the Be star population due to binary evolution: Cool giants and white dwarfs have been searched for with limited success. The main remaining possibility are hot sub-dwarfs. Interstellar HI would prevent their detection from the combined spectral energy distribution unless the Be star is so cool that the flux excess becomes visible at longer wavelengths. Other techniques are high-S/N observations of high-excitation lines forming in the region of the disk facing such a companion, radial-velocity measurements, and interferometry. If low-order NRP is indeed important for the Be phenomenon, searches for them in Be stars spun up in binary systems may make an important contribution to the unresolved issue of the homogeneity of the Be phenomenon. How do B stars spun up in binaries become Be stars if rapid rotation alone is not a sufficient condition?

- The disk or stellar origin of the winds of Be stars: Spectroscopic monitoring of optical disk emission lines and UV resonance lines over one or two disk life cycles in a small number of Be stars should clarify this issue.

- The disk-truncation mechanism in Be stars: The combination of spectro-interferometry at IR to mm wavelengths and models for the dynamics and radiative transfer in disks will give the answer.

- The nature(s) of B[e] stars: Parallaxes from GAIA will be an important step towards understanding the reported diversity. With some luck, objects observable with interferometers may be identified.

- The wind structure of $O B$ supergiants: Simultaneous UV and optical spectroscopy, spectroplarimetry, and IR and $\mathrm{mm}$ observations designed such as to permit discrimination between processes scaling with $\rho$ and $\rho^{2}$ will give important guidance to model calculations. If winds from Be stars do not originate from the photosphere but nevertheless share properties with the winds of more luminous stars, interpretative efforts may perhaps abstract more strongly from putative photospheric print-throughs.

- The origin of magnetic fields in radiative atmospheres: Seismological comparisons of magnetic and non-magnetic stars and observations in star clusters will constrain the structure and evolutionary phase of magnetic stars. But most of the load will be carried by detailed model calculations.

- The progenitors and descendants of Active OB Stars: This is the area, in which the Active OB Star community has the least autonomy. But it also offers a large opportunity for external impacts.

To almost all topics, observations of Active OB Stars in galaxies with different metallicities will make fundamental contributions, and accurate abundance analyzes will often yield important clues on the time-integrated evolutionary effects in individual stars.

Perhaps the most important steps forward can be expected from numerical model calculations. Simulations of many activities will be possible in three dimensions, and time dependencies will be included. It will be possible to weave extensive model grids even around specific sets of observations and thereby place tight limits on the derived quantitative parameters. Real interferometric images may be within reach.

$\mathrm{Un}($ ?)fortunately, there is no way to predict genuinely new discoveries. Massive Population III stars may be one of them. In any event, new developments will probably force the title of that future IAU Symposium to be: Active OB Stars: Ever new challenges.

\section{References}

On purpose, this paper does not provide any references: What is not expanded on in the following papers probably does not matter for the scope of this Symposium. 


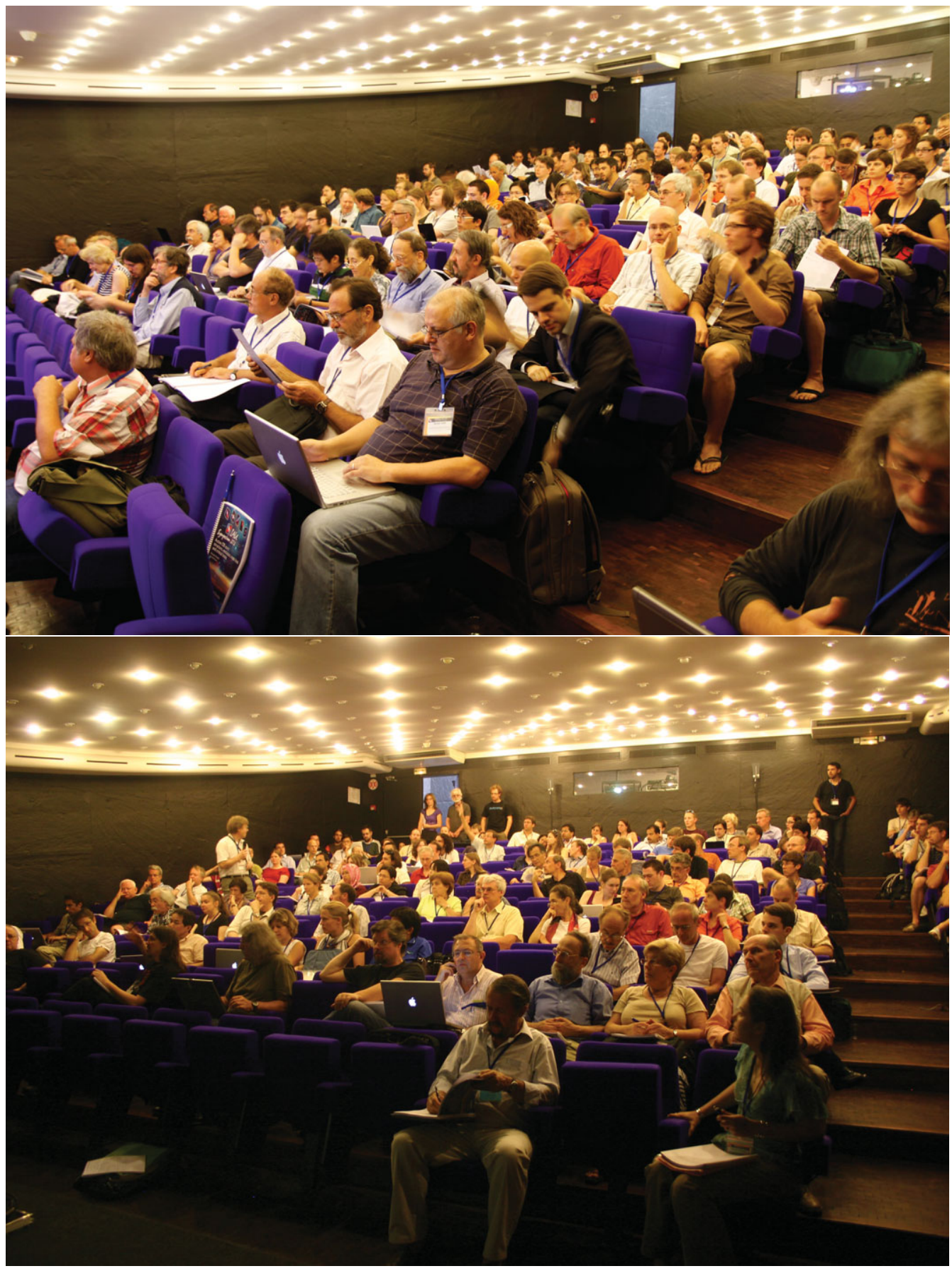

Participants in the auditorium. 